\title{
Gene expression of phosphorus transport and sugar metabolism in Medicago lupulina plants with inoculation by Rhizophagus irregularis under conditions of low phosphorus levels in the substrate
}

\author{
Yurkov A.P. ${ }^{1,2 *}$, Kryukov A.A. ${ }^{1}$, Gorbunova A.O. ${ }^{1,2}$, Dobryakova K.S. ${ }^{3}$, \\ Afonin A.M. ${ }^{1}$, Shishova M.F. ${ }^{2}$ \\ ${ }^{1}$ All-Russian Research Institute for Agricultural Microbiology, St. Petersburg, Russia \\ ${ }^{2}$ St. Petersburg State University, St. Petersburg, Russia \\ ${ }^{3}$ Komarov Botanical Institute, RAS, St. Petersburg, Russia \\ *e-mail: yurkovandrey@yandex.ru
}

The aim of this study is to assess the expression level of the main phosphorus transport genes and carbohydrate metabolism genes in highly mycotrophic line M1S-1 Medicago lupulina inoculated with highly efficient arbuscular mycorrhizal fungus Rhizophagus irregularis under conditions of low level of phosphorus available for plant nutrition in the substrate. In our work, we used the original method for estimating the expression of genes, the sequences of which were selected by homology with the known sequences for $M$. truncatula and according to the data of the transcriptome analysis for M. lupulina. In particular, carbohydrate metabolism genes (MlHXK1, MlSUS, MlSUS2, MlSUC4, $M l r b c S, M l S T P 13)$ and phosphorus transport (MlPT1, MlPT2, MlPT4, MlATP1) were detected. For each genes, three pairs of primers were selected, of which the most effective ones were left to assess relative expression using the generally accepted 2- $\Delta \Delta C \mathrm{CT}$ method (reference gene - actin). Accounting for the parameters of productivity, efficiency of arbuscular mycorrhiza (AM), mycorrhization, microelements content, and expression of genes of interest were carried out in the key stages of $M$. lupulina plant development (6 terms up to flowering stage). Pronounced dynamics were shown for all genes of interest except $M l H X K 1$ and MlRUB. The study showed that two genes - MlPT4, MlATP1 are specific for the development of effective AM-symbiosis of M. lupulina with the fungus R. irregularis. MlSUS2 showed significantly higher expression in plant roots with AM against control. Since 2019, it is planned to add several new genes to the analysis, such as invertase genes, as well as the MST or SWEET genes, among the latter screening for the presence of plant mycorrhiza-specific sugar transporters can be performed.

Acknowledgements: This work was supported by RFBR grant (No. 18-016-00220) and RSF grant (No. 16-16-00118). 